\title{
ANÁLISIS CRÍTICO COMPARATIVO DE PROSTATECTOMÍA RADICAL ABIERTA, LAPAROSCÓPICA Y ROBÓTICA: RESULTADOS DE MORBILIDAD PERIOPERATORIA Y CONTROL ONCOLÓGICO (PARTE I).
}

\author{
Juan I. Martínez-Salamanca' y Javier Romero Otero².
}

'Robotic Prostatectomy Program \& Urology Oncology Outcomes. Weill Medical College at Cornell University. New York Presbyterian Hospital. Brady Urological Institute. New York. USA.

${ }^{2}$ Department of Urology. Memorial Sloan-Kettering Cancer Center. New York. USA.

\begin{abstract}
Resumen.- OBJETIVO: El tratamiento quirúrgico del cáncer de próstata clínicamente localizado ha demostrado ser una opción excelente en lo que ha resultados oncológicos y morbilidad operatoria se refiere. Actualmente coexisten tres abordajes; abierto, laparoscópico y robótico. Debido a la experiencia acumulada y al largo seguimiento de las series, la prostatectomía radical abierta sigue siendo el "patrón oro". Pero, sin duda, los grupos laparoscópicos y robóticos van aportando una experiencia nada despreciable. En los próximos años debemos dilucidar a la vista de los resultados las ventajas e inconvenientes de cada una de ellas. En este trabajo hemos realizado una revisión de la literatura para intentar comparar las tres técnicas, centrándonos en la morbilidad quirúrgica y los resultados oncológicos disponibles.
\end{abstract}

MÉTODOS: Realizamos una búsqueda sistemática en las siguientes bases de datos: PubMed; EMBASE; Cochrane; SCOPUS; Science Citation Index durante el periodo enero de 1990 a enero de 2007 para los términos: "radical retropubic prostatectomy"; "open radical prostatectomy"; "laparoscopic prostatectomy"; "laparoscopic radical prostatectomy"; "robotic prostatectomy"; "robotic radical prostatectomy"; "treatment outcome"; "oncologic outcome"; "outcomes morbidity"; "mortality" y "minimally invasive treatment". Analizamos las series más representativas (curva de aprendizaje finalizada) para cada uno de los tres abordajes en cuanto a morbilidad perioperatoria y resultados oncológicos.

RESULTADOS: Existe una ausencia en la literatura de estudios aleatorizados para poder realizar una comparación objetiva y veraz de las tres técnicas. En este escenario, hemos analizado las series individuales más representativas. En cuanto a los resultados perioperatorios analizados, parecen no existir diferencias claras en cuanto a tiempo quirúrgico, complicaciones intraoperatorias, días de sondaje y estancia hospitalaria. Por el contrario, los abordajes endoscópicos llaparoscopia y robótical presentan unos resultados mas favorables en cuanto a menor pérdida sanguínea y menor necesidad de analgesia postoperatoria. Los resultados oncológicos a corto plazo (márgenes quirúrgicos) parecen ser comparables entre los tres abordajes. Sólo disponemos de datos a largo plazo (más de 10 años) sobre recurrencia bioquímica de las series abiertas.

CONCLUSIONES: La cirugía abierta sigue siendo el referente para todas las comparaciones. La cirugía laparoscópica esta siendo sustituida (sobre todo en Norteamérical por la cirugía robótica, que se encuentra en plena expansión. Es necesario, la publicación de estudios aleatorizados y prospectivos para poder comparar objetivamente las tres técnicas. 
Palabras clave: Cáncer de próstata. Prostatectomía abierta. Retropúbica. Laparoscópica. Robótica. Resultados.

Summary.- OBJECTIVES: With regard to oncological outcomes and perioperative morbidity, surgical treatment of localized prostate cancer has proved to be an excellent treatment option. At present, there are three different approaches, open, laparoscopic and robotic. Open radical prostatectomy remains the "gold Standard" due to accumulated experience and long follow up of series. But, without a doubt, laparoscopic and robotic groups are contributing with a far from negligible experience. Looking carefully at outcomes we will have to elucidate amongst advantages and disadvantages of each one of them. In this study we have performed a review of the literature trying to compare the three techniques, focusing in available surgical morbidity and oncological outcomes.

METHODS: We performed a systematic search in the following data bases: PubMed; EMBASE; Cochrane; SCOPUS; Science Citation Index, from January 1990 to January 2007 for terms: "radical retropubic prostatectomy"; "open radical prostatectomy"; "laparoscopic prostatectomy"; "laparoscopic radical prostatectomy"; "robotic prostatectomy"; "robotic radical prostatectomy"; "treatment outcome"; "oncologic outcome"; "outcomes morbidity"; "mortality" and "minimally invasive treatment". We analyzed the most representative series (finished learning curve) in each one of the three approaches regarding perioperative morbidity and oncological outcomes.

RESULTS: To be able to perform an objective and truthful comparison of the three techniques, there is an absence in randomized studies in the literature. In this scenario we have analyzed the most representative individual series. With respect to the analyzed perioperative results, it seems to be no clear differences regarding surgical time, intraoperative complications, and catheter and hospital stay days. Otherwise, the endoscopic approach (laparoscopy and robotics) show a more favorable results in respect of blood loss and less postoperatorive analgesic. The shortterm oncological outcomes Isurgical margins) seem to be comparable amongst the three approaches. We only have long-term data (more than 10 years) on open series biochemical recurrence.

CONCLUSIONS: The open surgery has become a reference for all comparisons. The laparoscopic surgery is being replaced (specially in North America) by robotic surgery which is constantly expanding. It is necessary the publication of randomized and prospective studies to be able to objectively compare the three techniques.

Keywords: Prostate cancer. Radical prostatectomy. Laparoscopic radical prostatectomy. Robotic radical prostatectomy. Morbidity.

\section{INTRODUCCIÓN}

La Prostatectomía Radical (PR) es una de las opciones terapéuticas más extendidas como tratamiento del cáncer de próstata localizado. La PR ha sido una técnica quirúrgica en constante desarrollo. Fue descrita en el siglo pasado por Young (1) a través de un abordaje perineal. Posteriormente adaptada por Millin (2) para desarrollarla vía retropúbica, ofreciendo la posibilidad de realizar linfadenectomía mediante una única incisión. El desarrollo de la técnica fue progresivo hasta que se consiguió un control oncológico excelente pero siempre a expensas de una mutilación funcional importante, que se traducía en tasas de incontinencia e impotencia muy altas (3-6). Fueron Walsh y Donker (7) quienes en 1982 establecieron las bases anatómicas y quirúrgicas para la preservación de los haces neurovasculares durante la PR. Este hecho cambió el objetivo de esta cirugía, de control oncológico y mínima morbilidad; a control oncológico, preservando la continencia y erección, con la mínima morbilidad (8).

Desde entonces todos los esfuerzos se han dirigido al desarrollo de técnicas que sin poner en peligro el control de la enfermedad y manteniendo los buenos resultados iniciales, fuesen lo menos invasivas posibles y tratasen de mejorar los resultados funcionales. Con este espíritu Raboy y cols. en 1997 (9) publicaron la realización de la primera prostatectomía radical laparoscópica (PRL). Ese mismo año Schuessler y cols. (10) comunicaron la primera serie de PRL, pero en su experiencia no aportaba ninguna ventaja sobre la prostatectomía radical abierta (PRA). Fue el grupo de Guillonneau y cols. (11) en 1998 quienes describieron la PRL como un procedimiento factible y que podía ser considerado como un abordaje más para esta cirugía. En la continua lucha por mejorar los resultados y hacer más accesible esta técnica ha aterrizado la prostatectomía radical robótica(12) (PRR), que es ya una realidad en Estados Unidos y comienza a serlo en Europa. No obstante la PRA continúa siendo la técnica quirúrgica con la que los nuevos abordajes deben compararse.

Desde la introducción por Wang y cols. (13) en 1979 del PSA y su uso masivo en la práctica clínica para la detección del cáncer de próstata se ha producido una migración en el estadio clínico al diagnóstico (14). En la actualidad se tratan tumores de menor agresividad, con excelente control oncológico a corto y largo plazo (15-17), que hacen que los resultados funcionales comiencen a ser una de las variables más consideradas a la hora de decidir una opción terapéutica. 
En esta serie de dos artículos se ha realizado una revisión sistemática de la literatura para conocer los resultados perioperatorios, oncológicos y funcionales de cada una de las tres técnicas (Prostatectomia Radical Abierta (PRA), Prostatectomía Radical Laparoscópica (PRL) y Prostatectomía Radical Robótica (PRR)). La ausencia de estudios prospectivos y aleatorizados que aporten evidencia científica en la comparación nos debemos ceñir a la descripción de los resultados de las series más amplias y significativas.

El esquema de desarrollo ha sido el siguiente:

\section{- Morbilidad Perioperatoria}

- Tiempo quirúrgico

- Pérdida sanguínea

- Complicaciones intraoperatorias

- Días de sondaje y anastomosis vesicouretral

- Analgesia postoperatoria

- Estancia hospitalaria
- Control Oncológico

Márgenes quirúrgicos

Control bioquímico (Progresión de PSA)

\section{- Continencia Urinaria}

- Disfunción Eréctil

\section{MATERIAL Y MÉTODOS}

Realizamos una búsqueda sistemática en: PubMed; EMBASE; Cochrane; SCOPUS; Science Citation Index durante el período de Enerol990 a Enero 2007 para los términos: "radical retropubic prostatectomy"; "open radical prostatectomy"; "laparoscopic prostatectomy"; "laparoscopic radical prostatectomy"; "robotic prostatectomy"; "robotic radical prostatectomy"; "treatment outcome"; "oncologic outcome"; "outcomes morbidity"; "mortality" y "minimally invasive treatment". Analizamos las series

TABLA I. DATOS PERIOOPERATORIOS (I).

\begin{tabular}{|c|c|c|c|c|c|c|}
\hline Autor & Año & $\begin{array}{c}\text { № } \\
\text { Casos }\end{array}$ & $\begin{array}{c}\text { Tiempo } \\
\text { Quirúrgico } \\
\text { (min) }\end{array}$ & $\begin{array}{c}\text { Pérdida } \\
\text { Sanguínea } \\
\text { (ml) }\end{array}$ & $\begin{array}{c}\text { Transfusión } \\
\text { (\%) }\end{array}$ & $\begin{array}{c}\text { Reconversión } \\
(\%)\end{array}$ \\
\hline \multicolumn{7}{|c|}{ PROSTATECTOMÍA RADICAL ABIERTA } \\
\hline Lepor (59) & 2003 & 1024 & 129 & 805 & 8.2 & SD \\
\hline Salomón (60) & 2002 & 145 & 197 & SD & 26.2 & SD \\
\hline Lerner (61) & 1995 & 1000 & SD & SD & 12 & SD \\
\hline \multicolumn{7}{|c|}{ PROSTATECTOMÍA RADICAL LAPAROSCÓPICA } \\
\hline Guillonneau (62) & 2001 & 240 & 239 & 402 & 10 & 2.5 \\
\hline Rassweiler (63) & 2003 & 219 & 288 & 1100 & 30.1 & 4.2 \\
\hline Abbou (60) & 2000 & 137 & 285 & SD & 2.9 & SD \\
\hline \multicolumn{7}{|c|}{ PROSTATECTOMÍA RADICAL ROBÓTICA } \\
\hline Menon (64) & 2004 & 1100 & 160 & $50-250$ & $0 \%$ & $0 \%$ \\
\hline $\mathrm{Hu}(65)$ & 2006 & 322 & 186 & 250 & $1.6 \%$ & $0.6 \%$ \\
\hline Farnham-Smith (27) & 2006 & 176 & SD & 191 & $0.5 \%$ & $0 \%$ \\
\hline Joseph (66) & 2006 & 325 & 130 & 196 & $1 \%$ & $0 \%$ \\
\hline Van Applendorn-Costello (67) & 2006 & 150 & 191 & SD & $2.6 \%$ & $0 \%$ \\
\hline
\end{tabular}


más representativas (curva de aprendizaje finalizada) para cada uno de los tres abordajes en cuanto a morbilidad perioperatoria y resultados oncológicos.

\section{MORBILIDAD PERIOPERATORIA}

\section{- TIEMPO QUIRÚRGICO}

La duración de la intervención ha sido uno de los grandes caballos de batalla del abordaje mínimamente invasivo, tanto laparoscópico como robótico. Los tiempo iniciales de las series laparoscópicas eran tremendamente largos (18-21) aunque fueron mejorando a medida que avanzaba en la curva de aprendizaje $(22,23)$. Con la cirugía robótica está sucediendo algo parecido, y los trabajos demuestran que el tiempo quirúrgico se acorta de manera significativa y exponencial cuando aumenta el número de casos (24). Los tiempos quirúrgicos en el inicio de la curva de aprendizaje robótica, parecen algo inferiores (Tabla I) a los comunicados inicialmente por los grupos de laparoscopia y muy similares a los tiempos medios de PRA de las series recientes.

Sin duda la cirugía robótica se ha beneficiado de la reducción de tiempos al reproducir una técnica bien descrita y conseguida con el abordaje laparoscópico clásico. Las demoras en los tiempos iniciales de las series robóticas, se relacionaron con el tiempo para acoplar el dispositivo. Muchos centros de excelencia han conseguido disminuir este tiempo debido a la práctica diaria y sobre todo a tener un equipo humano fijo y perfectamente adiestrado para esta tarea (25).

En resumen, en manos experimentadas los tiempos tienden a igualarse con cualquier abordaje y en cualquier caso, las diferencias no parecen ser tan importantes como para impactar en la morbilidad peri operatoria.

\section{- PÉRDIDA SANGUIINEA}

La mayoría de las publicaciones muestran una menor pérdida sanguínea intraperatoria con abordajes laparoscópicos (con o sin robot). La presión intraabdominal facilitada por el neumoperitoneo, colapsa vasos sanguíneos, fundamentalmente venosos, que son una de las fuentes más importantes de hemorragia durante la prostatectomía. Asimismo, en las técnicas endoscópicas, la sección del complejo venoso dorsal, se realiza al final de la intervención, una vez que la próstata esta prácticamente extirpada. Son varias las series que comunican pérdidas menores a 100 o $200 \mathrm{ml}(22,26-28)$.

Farnham y cols. han publicado recientemente una comparación prospectiva, entre PRA Y PRR en términos de pérdida sanguínea. Sus resultados fueron, que la media de hematocrito en el momento del alta fue de $38 \%$ tras PRR y de $33 \%$ tras PRA.

La diferencia final, la marca la necesidad o no de transfusión de hemoderivados. Como se refleja en la Tabla I, las cifras de transfusión son sensiblemente superiores en la series de cirugía abierta.

Dos experiencias muy amplias (mas de 2000 pacientes) publicadas recientemente $(24,29)$ una de PRL y otra de PRR comunican pérdidas sanguíneas mínimas (mediana < a 200 cc.) y cifras de transfusión intraoperatoria del 0\%. Quizás sean datos demasiado optimistas.

El impacto de la transfusión sanguínea mas allá de consideraciones religiosas o personales (teórico riesgo de contagio) puede tener impacto en el tiempo de reincorporación laboral así como en la demora para iniciar actividad física tras la intervención (30).

Otra posible reflexión importante en relación a la pérdida sanguínea, se basa en la posibilidad de disección mas precisa en ausencia de hemorragia importante. Esto puede tener impacto en la calidad de la preservación neurovascular, ya que muchas veces el cirujano se ve obligado a cambiar su plan de preservación uni o bilateral preoperatorio, debido al stress que genera la excesiva pérdida de sangre. Este hecho no esta estudiado en la literatura pero parece tener sentido.

De cualquier manera, realizar una comparación objetiva no es sencillo, ya que existe una gran diferencia de criterios entre los diferentes trabajos publicados. Por ejemplo, hay cirujanos que utilizan un nivel predeterminado de hemoglobina en sangre para trasfundir, independientemente del estado del paciente. Otros, por el contrario, esperan a que el paciente presente síntomas. Por tanto, existe un sesgo en la comparación, basado en la práctica clínica individual.

\section{- COMPLICACIONES INTRAOPERATORIAS}

Las lesiones intraoperatorias mas frecuentes comunicadas en las diferentes series son las siguientes:

- Lesión rectal.

- Lesión ureteral.

- Lesiones de grandes vasos (iliacos externos) durante la linfadenectomía.

Además de la necesidad de la detección inmediata y reparación, la aparición de estas lesio- 
nes puede conllevar a la necesidad de reconvertir el procedimiento (de PRR o PRL a PRA o de PRR a PRL - PRA). Las series más importantes y con curva de aprendizaje finalizada reflejan unas cifras muy bajas tanto de lesiones como de necesidad de reconversión (Tabla II).

Rassweiler y cols. en un estudio multiintitucional encontraron que las causas mas frecuentes de reconversión del procedimiento fueron; adherencias, dificultades para realizar la anastomosis, mal función de los instrumentos y riesgo de margen positivo por la extensión o localización del tumor (31).

No cabe duda que los factores más importantes para evitar estas lesiones son la buena indicación del procedimiento y la experiencia del cirujano. No parece que la técnica en si misma, tenga por que tener impacto en unas mayores cifras de complicaciones durante la cirugía.

A favor de la PRR, debemos decir que al haber demostrado una curva de aprendizaje mucho menor que la PRL, esto tiene impacto positivo en el número de complicaciones intraoperatorias durante, al menos, la curva de aprendizaje $(32,33)$.

\section{- DIAS DE SONDAJE Y ANASTOMOSIS VESI- COURETRAL}

Las series clásicas de prostatectomía abierta, recomendaban entre 2 y 3 semanas de sonda (34). A medida que el refinamiento en la técnica, ha permitido hacer una anastomosis de mejor calidad, los días de sondaje han ido disminuyendo, hasta 7-10 días. (Tabla II) Las grandes series de PRL, mantienen la sonda entre 5 y 7 días.

TABLA II. DATOS PERIOOPERATORIOS (II).

\begin{tabular}{|c|c|c|c|c|}
\hline Autor & $\begin{array}{c}\text { Compli- } \\
\text { caciones } \\
(\%)\end{array}$ & Complicaciones mayores & $\begin{array}{c}\text { Estancia } \\
\text { (días) }\end{array}$ & $\begin{array}{c}\text { Sondaje } \\
\text { (días) }\end{array}$ \\
\hline \multicolumn{5}{|c|}{ PROSTATECTOMÍA RADICAL ABIERTA } \\
\hline Lepor (59) & 6.2 & SD & 2.3 & $7-10$ \\
\hline Salomon (60) & 16.5 & Lesión rectal(4),TVP(6), TEP(1) & 15.2 & 15.9 \\
\hline Catalona (68) & 10 & $\operatorname{TEP}(2), \operatorname{IAM}(2), \mathrm{HPE}(11)$, Lesión rectal (1) & SD & SD \\
\hline \multicolumn{5}{|c|}{ PROSTATECTOMÍA RADICAL LAPAROSCÓPICA } \\
\hline Rassweiler $(31,69)$ & 13.9 & Lesión Rectal (1.2),TEP (0.3\%), IAM (0.1\%) & SD & 7 \\
\hline Abbou (60) & 13.1 & $\operatorname{TVP}(1), \operatorname{TEP}(1), \mathrm{PNA}(1)$, lesión Rectal(2),íleo (4), & 7.4 & 6.8 \\
\hline Guillonneau $(70,71)$ & 0.7 & $\begin{array}{l}\text { Lesión ureteral( } 1) \\
\text { Lesión intestinal }(1.2 \%) \text {, Lesión ureteral }(0.8 \%)\end{array}$ & 6 & 6.6 \\
\hline \multicolumn{5}{|c|}{ PROSTATECTOMÍA RADICAL ROBÓTICA } \\
\hline Menon (64) & $5 \%$ & TVP (1), Lesión intestinal (2), edema pulmonar (1), HPE (2) & 1.2 & 7 \\
\hline $\mathrm{Hu}(65)$ & $17.2 \%$ & $\begin{array}{l}\text { Lesión ureteral (1), FU (24), estenosis cuello vesical (2), } \\
\text { íleo (9), hemorragia (2), TVP (2) }\end{array}$ & SD & SD \\
\hline Joseph (66) & $9.6 \%$ & $\begin{array}{l}\text { FU (4), Lesión rectal (1), estenosis cuello vesical (7), TVP } \\
(4), \text { IAM (3), TEP (1) }\end{array}$ & 1 & SD \\
\hline $\begin{array}{l}\text { Van Applendorn- } \\
\text { Costello (67) }\end{array}$ & $3.3 \%$ & Lesión rectal (1), hemorragia (4) & 3.4 & SD \\
\hline
\end{tabular}

SD: Sin datos TVP: Trombosis Venosa Profunda, HPE: Hernia Puerto de Entrada o Incisional, TEP:Tromboembolismo Pulmonar, FU: Fístula Urinaria, IAM: Infarto Agudo de Miocardio. 
La mejor visualización que proporcionan las técnicas laparoscópicas y fundamentalmente la robótica (magnificación e imagen tridimensional), están permitiendo realizar, en manos experimentadas, una anastomosis uretro-vesical de alta calidad. Aunque no se debe olvidar, que existe un componente de edema postoperatorio, asociado intrínsecamente a la agresión quirúrgica y que necesita un tiempo mínimo para resolverse.

Algunos grupos laparoscópicos han propuesto retirar la sonda precozmente (2-3 días), pero comunicando un $11 \%$ de retenciones urinarias (35).

Menon y cols. han comunicado recientemente su proceder tras más de 2500 procedimientos rea- lizados (PRR) (24). Mantienen la sonda de 4 a 7 días y la retiran previa cistografía.

Una vez más, es difícil poder evaluar con que técnica se podrían disminuir los días de sondaje, sin aumentar el número de fístulas urinarias clínicas y sin impacto en los resultados de continencia a medio y largo plazo.

\section{- ANALGESIA POSTOPERATORIA}

Cualquiera de los tres abordajes confiere al paciente un postoperatorio razonable, en relación al dolor.

Algunos estudios comparativos muestran una diferencia clara entre los abordajes laparoscópicos o

TABLA III. RESULTADOS PATOLÓGICOS Y SOBRE MÁRGENES QUIRÚRGICOS.

\begin{tabular}{|c|c|c|c|c|c|c|c|c|c|}
\hline \multirow[t]{2}{*}{ Autor/Año } & \multirow[t]{2}{*}{ Periodo } & \multirow[t]{2}{*}{ Casos } & \multicolumn{3}{|c|}{ PTNM\% } & \multirow{2}{*}{$\begin{array}{l}\text { Tasa Global } \\
\text { MQP (\%) }\end{array}$} & \multicolumn{3}{|c|}{ MQP\% } \\
\hline & & & pT2 & pT3a & pT3b & & pT2 & pT3a & pT3b \\
\hline \multicolumn{10}{|c|}{ PROSTATECTOMÍA RADICAL ABIERTA } \\
\hline Catalona (1998) (72) & $83-97$ & 1778 & SD & \multicolumn{2}{|c|}{ SD } & 20.9 & SD & \multicolumn{2}{|c|}{ SD } \\
\hline Huland (2001) (73) & SD & 789 & SD & \multicolumn{2}{|c|}{ SD } & SD & 14.9 & \multicolumn{2}{|c|}{36.5} \\
\hline Salomon (2002) (60) & $88-00$ & 145 & 62.1 & 17.2 & 20.6 & SD & 18.8 & \multicolumn{2}{|c|}{52.7} \\
\hline Lepor (2003) (74) & $94-00$ & 1000 & SD & \multicolumn{2}{|c|}{ SD } & 19.9 & SD & \multicolumn{2}{|c|}{ SD } \\
\hline \multicolumn{10}{|c|}{ PROSTATECTOMÍA RADICAL LAPAROSCÓPICA } \\
\hline Abbou (2000) (60) & $88-00$ & 137 & 75.9 & 13.8 & 10.2 & 28.4 & 21.9 & \multicolumn{2}{|c|}{40.8} \\
\hline Rassweiler (2005) (75) & $99-04$ & 1078 & 59.2 & 21.4 & 17.6 & SD & 7.4 & \multicolumn{2}{|c|}{31.8} \\
\hline Guillonneau (2003) (55) & $98-02$ & 1000 & SD & \multicolumn{2}{|c|}{$\mathrm{SD}$} & SD & 15.5 & \multicolumn{2}{|c|}{31.1} \\
\hline Gaston (2006) (29) & 03-05 & 413 & 58.5 & 35.5 & 5.8 & 30.7 & 21.9 & 43 & 46 \\
\hline \multicolumn{10}{|c|}{ PROSTATECTOMÍA RADICAL ROBÓTICA } \\
\hline Patel (2005) (57) & 03-04 & 200 & 78 & 14 & 8 & 10.5 & 5.7 & 26.2 & 33 \\
\hline Chien (2005) (76) & 03-04 & 56 & 82 & \multicolumn{2}{|c|}{18} & 11 & SD & SD & SD \\
\hline $\mathrm{Hu}(2006)(65)$ & 03-04 & 322 & 24.8 & 0.3 & 0 & SD & SD & SD & SD \\
\hline Joseph (2006) (66) & $05-06$ & 325 & 81 & 14 & 5 & 13 & 9.9 & 37.1 & 27.3 \\
\hline $\begin{array}{l}\text { Van Applendorn-Costello } \\
\text { (2006) (67) }\end{array}$ & $03-04$ & 150 & SD & SD & SD & 17.3 & SD & SD & SD \\
\hline Menon (2007) (24) & $01-06$ & 1142 & 77.7 & 16.9 & 5.1 & 13 & SD & SD & S \\
\hline
\end{tabular}


robóticos y los abiertos, a favor de los primeros, en cuanto a menos dolor postoperatorio $(26,36)$. Recientemente el grupo de Vanderbilt, ha publicado un trabajo dentro de su propia institución y prospectivo, en el cual no se observan diferencias estadísticamente significativas entre ambos grupos, básicamente debido, a los bajos índices de dolor con ambas técnicas (PRA y PRR) (37).

Las incisiones cutáneas son muy similares en la PRL y PRR y producen mínimo dolor postoperatorio. Por otro lado, la incisión que se realiza en la PRA (laparotomía media infraumbilical) causa poca morbilidad, al no seccionar vientres musculares. Por tanto, la teórica ventaja en la disminución del dolor postoperatorio, no es tan clara como en otros escenarios (p.e. cirugía renal).

\section{- ESTANCIA HOSPITALARIA}

En líneas generales la optimización de la técnica de prostatectomía, ha reducido de manera determinante la estancia posquirúrgica en las últimas décadas. La mayoría de los grupos tanto robóticos como laparoscópicos tienen un ingreso, en la mayoría de los casos, de entre 24 y 48 horas $(26,38)$. El motivo que habitualmente alarga el ingreso, es el íleo paralítico que provoca intolerancia digestiva.

Las series de PRA y PR Perineal señalan ingresos algo superiores, aunque son muy comunes los programas actuales, con estancia reducida. (Tabla III).

De cualquier manera, se debe ser cauto a la hora de interpretar los tiempos de ingreso. Ya que, cada profesional debe adaptar esto, a cada paciente, a cada entorno socio-sanitario y a su propio juicio clínico. En sistemas sanitarios, con una presión excesiva en las cifras de estancia, las expectativas creadas en el paciente antes de la cirugía, tienen una importancia capital, en su propia decisión del alta hospitalaria.

\section{CONTROL ONCOLÓGICO}

Sin menospreciar los resultados funcionales, el primer objetivo en el tratamiento del cáncer de próstata localizado debe ser el control oncológico. Este control, lo podemos objetivar de dos maneras:

- Resultado inmediato tras la prostatectomía (primer valor de PSA y estado de los márgenes quirúrgicos de resección).

- Control a medio y largo plazo (ausencia de recidiva bioquímica y/o progresión).

Al no existir seguimiento suficiente (sobre todo en las series de PRL y PRR), los resultados de los márgenes quirúrgicos tras prostatectomía radical tienen un renovado interés como marcador de control oncológico.

\section{- MARGENES QUIRÚRGICOS}

Aunque el verdadero impacto del margen quirúrgico positivo (MQP) es aún controvertido, muchos autores están de acuerdo en que es un indicador independiente de mal pronóstico y puede en última instancia, relacionarse con una probabilidad mayor de recidiva bioquímica y disminución de la supervivencia cáncer-específica (39-44). El riesgo de fallo bioquímico a los 5 años por MQP varía entre el $42 \%$ al $64 \%$, que es significativamente más alto que en los pacientes con márgenes quirúrgicos negativos (43). Scardino y cols. revisaron recientemente su experiencia con 1389 PRR, estadio clínico T1-T3 (41). La cifra global de MQP, fue de $12.9 \%$, $6.8 \%$ para T2 y $23 \%$ para T3. Tras un seguimiento medio de 50 meses, la probabilidad de supervivencia libre de recidiva bioquímica fue del $58 \%$ en pacientes con márgenes negativos vs. $81 \%$ para pacientes con MQP. Esto se traduce un riesgo relativo para pacientes con MQP de 1.2-2.7. Estos autores concluyeron que un margen positivo incrementa el riesgo de recurrencia incluso después de ajustar los factores concurrentes de riesgo (PSA pre tratamiento, Gleason, estadio clínico y patológico). En otro gran estudio de 2500 PRA, Blute y cols. manifestaron similares hallazgos (44).

Las series históricas de PRA comunican unas cifras de MPQ discretamente superiores a las actuales con cualquiera de las tres técnicas. Las series actuales de PRA arrojan unas cifras de MPQ globales del 6.9 al $29 \%$. Para la PRL y PRR las cifras de las series más recientes son parecidas (entre el 5.7 y el $37.1 \%$ ) (Tabla III).

En su revisión de la literatura, Wieder y Soloway informaron de una amplia escala de MQP en las series de PRR, desde el $0 \%$ al $77 \%$, con un promedio del $36 \%$ (42). Por estadio los MQP fueron del 17\% en $\mathrm{T} 2 \mathrm{a}, 36 \%$ en $\mathrm{T} 2 \mathrm{~b}$, y $53 \%$ en $\mathrm{T} 3$.

Brown y cols. demuestra que no existen diferencias significativas relacionadas con la técnica en relación al número de MP entre PRA y PRL y entre PRA y PRR (45). En todos los grupos la zona mas frecuentes de MP es la posterolateral. Es bien conocido que la existencia de MP es un factor independiente de progresión bioquímica y que impacta de manera negativa en la supervivencia (46-48).

Independientemente del tipo de abordaje, el número de MQP ha disminuido con el paso del tiempo debido a la migración de estadio en la era del PSA. 
Se aconseja cautela cuando se interpretan los márgenes quirúrgicos entre series, ya que son muy subjetivos y no están estandarizados entre las instituciones. Adicionalmente, algunos autores informan del estado del márgen basado en biopsias intraoperatorias y no el borde de tinta china de la pieza $(12,49)$. El estado de los márgenes quirúrgicos se ve también afectado por el estadio clínico, PSA sérico y Gleason previo (50). Cuando se estratificó por nivel de PSA, los investigadores en el Hospital Henri Mondor informaron de márgenes positivos en el $20.6 \%$ de los pacientes con PSA $<10 \mathrm{ng} / \mathrm{ml}$, versus $25 \%$ en el total de la serie $(51,52)$. De forma similar, cuando se consideraron los pacientes con características favorables (PSA $<10 \mathrm{ng} / \mathrm{ml}$, enfermedad cTlc, y escala de Gleason $\leq 7$ ), Ahlering y cols. mostraron una disminución en el conjunto de márgenes positivos del $36 \%$ al $17 \%$ (53). En resumen las comparaciones de los MQP deberían realizarse únicamente después de ajustar las covariables pertinentes.

- CONTROL BIOQUÍMICO (PROGRESIÓN DE PSA)

La PRA has demostrado tras más de 15 años unos resultados excelentes, en relación al control oncológico (46-48). Los datos a este respecto son limitados en cuanto a seguimiento en la PRL y más aún en la PRR.

En la serie de la Clínica Mayo de PRA, entre 1998 y 2003 , las cifras del número de pacientes libres de progresión bioquímica a 3 y 5 años fueron del $99 \%$ y $98 \%$ respectivamente (54). Guillonneau y cols. evaluaron los resultados de un total de 1000 pacientes sometidos a PRL encontrando unos resultados

TABLA IV. RESULTADOS SOBRE CONTROL BIOQUÍMICO.

\begin{tabular}{|c|c|c|c|c|}
\hline \multirow[t]{2}{*}{ Autor } & \multirow[t]{2}{*}{ Período } & \multirow[t]{2}{*}{ № Pacientes } & \multicolumn{2}{|c|}{ \%Libre de recidiva } \\
\hline & & & 5 años & 10 años \\
\hline \multicolumn{5}{|c|}{ PROSTATECTOMÍA RADICAL ABIERTA } \\
\hline Han (16) & $82-99$ & 2494 & 92 & 85 \\
\hline Catalona (72) & $83-97$ & 925 & 78 & 64 \\
\hline Zincke (47) & $89-92$ & 1000 & 70 & 52 \\
\hline Hull (61) & $83-98$ & 987 & 78 & 75 \\
\hline Bianco (39) & $83-03$ & 1746 & 82 & 77 \\
\hline Saranchuk (17) & $98-03$ & 647 & 88 & SD \\
\hline \multicolumn{5}{|c|}{ PROSTATECTOMÍA RADICAL LAPAROSCÓPICA } \\
\hline Curto (29) & $03-05$ & 413 & \multicolumn{2}{|c|}{11 meses $=93.5$} \\
\hline German Group (63) & $99-04$ & 5824 & \multicolumn{2}{|c|}{24 meses $=91$} \\
\hline Rassweiler (75) & $99-04$ & 500 & \multicolumn{2}{|c|}{20 meses $=89$} \\
\hline Rozet $(56)$ & $98-04$ & 600 & \multicolumn{2}{|c|}{12 meses $=95$} \\
\hline Guillonneau (55) & $98-02$ & 1000 & \multicolumn{2}{|c|}{36 meses $=90$} \\
\hline \multicolumn{5}{|c|}{ PROSTATECTOMIAA RADICAL ROBÓTICA } \\
\hline Menon (64) & $01-06$ & 1142 & \multicolumn{2}{|c|}{36 meses $=98$} \\
\hline Mikhail (58) & $05-06$ & 100 & \multicolumn{2}{|c|}{22 meses $=96$} \\
\hline Ahlering (75) & 03-04 & 50 & \multicolumn{2}{|c|}{20 meses $=82$} \\
\hline
\end{tabular}


de tiempo de supervivencia libre de progresión en relación al estadio patológico de $92 \%$ (pT2a), 88\% (pT2b), 77\% (pT3a) y $44 \%$ (pT3b) respectivamente (55). Mas recientemente, Rozet y cols. publicaron en su serie unas cifras de supervivencia libre de recidiva bioquímica del 95\% a 12 meses (56) (Tabla IV).

En cuanto a las series de PRR, aún debemos esperar mas tiempo, pero resultados iniciales como los comunicados por Patel y cols. sobre un total de 200 pacientes con 9.7 meses de seguimiento $195 \%$ supervivencia libre de recidiva bioquímica) apuntan a que podrían ser semejantes (57) (Tabla IV).

De forma similar, Mikhail y cols. informaron de un $96 \%$ de supervivencia libre de recidiva bioquímica en 100 pacientes con una media de seguimiento de 12 meses (58). Los resultados con un seguimiento mayor han sido publicados por Menon y cols. (24). De un total de 1142 pacientes, 26 (2.3\%) presentaron recidiva bioquímica, con una media de seguimiento de 36 meses (rango 12-66). El índice de recidiva bioquímica proyectado a 5 años, se calculó que sería de un $8.4 \%$. Aunque prometedores, estos resultados son aún muy iniciales (Tabla IV).

En resumen, las tres técnicas parecen razonablemente comparables en su eficacia para erradicar la enfermedad local, pero es necesario un seguimiento mayor para poder compararlas en cuanto a supervivencia libre de progresión.

\section{CONCLUSIONES}

Esta claro que la PRR contiene las ventajas originarias de la PRL, reduce la pérdida sanguínea y el dolor postoperatorio en comparación con la técnica abierta. Esto se traduce en la posibilidad de un alta hospitalaria precoz (24 horas). En la mayoría de las series de PRL, la estancia postoperatoria es de varios días en un tercio de los pacientes. Una posible explicación a este hecho puede ser, que con la laparoscopia convencional los tiempos quirúrgicos iniciales son mayores y esto impacta de manera clara en la agresión a la que se somete al paciente. No hay que olvidar, que muchos sistemas sanitarios no incentivan al profesional para que realice un alta precoz y tampoco los pacientes lo demandan. Incluso hay que centros que mantienen ingresados los pacientes hasta que retiran la sonda vesical. En la Tabla I se exponen los resultados de las series publicadas que consideramos han superado su curva de aprendizaje.

Varios estudios han demostrado unos buenos resultados en términos de control oncológico de la PRL. Sin embargo, por ejemplo en pacientes de alto riesgo de tener márgenes positivos (T3c) las series no arrojan una diferencia significativa entre cirugía abierta o cirugía laparoscópica. Menon y cols. ha publicado resultados oncológicos muy optimistas en su serie de PRR, pero como mencionamos anteriormente, ha sido severamente criticado por la comunidad oncológica por procesamientos no convencionales de las piezas quirúrgicas y los datos de supervivencia carece de madurez. Esta práctica no convencional hace difícilmente comparable los resultados de la PRR con los de la cirugía abierta.

Las cifras de reconversión en la series de PRL son menores al $5 \%$. En la actualidad, las series más recientes de PRR refieren la mayoría de ellas $0 \%$ de reconversiones y otras cifras muy bajas. Las complicaciones perioperatorias en general son similares a las acaecidas durante cirugía laparoscópica, aunque la curva de aprendizaje y el tiempo operatorio son claramente favorables a la cirugía robótica.

Quizás el reto ahora sea demostrar la utilidad de la magnificación y la visión tridimensional que proporciona la robótica para intentar mejorar estos datos. En líneas generales, los resultados oncológicos de las series robóticas publicadas son equivalentes a las series clásicas de cirugía abierta o laparoscópica.

\section{BIBLIOGRAFÍA y LECTURAS

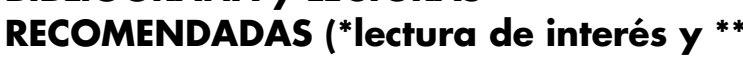 lectura fundamental)}

1. YOUNG, H.: "The early diagnosis and radical cure of carcinoma of the prostate". Bull Hopkins University, 175: 315, 1905.

2. MILLIN, T.M.D.: "Retropubic prostatectomy: a new extravesical technique". Lancet, 693, 1945.

3. JEWETT, H.J.: "Treatment of early cancer of the prostate". Jama, 183: 373, 1963.

4. KOPECKY, A.A.; LASKOWSKI, T.Z.; SCOTT, R. Jr.: "Radical retropubic prostatectomy in the treatment of prostatic carcinoma". J. Urol., 103: 641, 1970.

5. VEENEMA, R.J.; GURSEL, E.O.; LATTIMER, J.K.: "Radical retropubic prostatectomy for cancer: a 20-year experience”. J. Urol., 117: 330, 1977.

6. CORREA, R.J. Jr. y cols.: "Total prostatectomy for stage B carcinoma of the prostate". J. Urol., 117: 328, 1977.

7. WALSH, P.C.; DONKER, P.J.: "Impotence following radical prostatectomy: insight into etiology and prevention". J. Urol., 128: 492, 1982.

8. WALSH, P.C.: "The status of radical prostatectomy in the United States in 1993: where do we go from here?". J. Urol., 152: 1816, 1984. 
9. RABOY, A.; FERZLI, G.; ALBERT, P.: "Initial experience with extraperitoneal endoscopic radical retropubic prostatectomy". Urology, 50: 849, 1997.

10. SCHUESSLER, W.W. y cols.: "Laparoscopic radical prostatectomy: initial short-term experience". Urology, 50: 854, 1997.

11. GUILLONNEAU, B. y cols.: "Laparoscopic radical prostatectomy. Preliminary evaluation after 28 interventions". Presse Med., 27: 1570, 1998.

12. MENON, M.; TEWARI, A.: "Robotic radical prostatectomy and the Vattikuti Urology Institute technique: an interim analysis of results and technical points". Urology, 61: 15, 2003.

13. WANG, M.C. y cols.: "Purification of a human prostate specific antigen". Invest. Urol., 17: 159, 1979.

14. GRUBB, R.L. y cols.: "Minimally invasive approaches to localized prostate carcinoma". Hematol. Oncol. Clin. North Am., 20: 879, 2006.

15. HULL, G.W. y cols.: "Cancer control with radical prostatectomy alone in 1,000 consecutive patients". J. Urol., 167: 528, 2002.

16. HAN, M. y cols.: "Long-term biochemical diseasefree and cancer-specific survival following anatomic radical retropubic prostatectomy. The 15 -year Johns Hopkins experience". Urol. Clin. North Am., 28: 555, 2001.

17. SARANCHUK, J.W. y cols.: "Achieving optimal outcomes after radical prostatectomy". J. Clin. Oncol., 23: 4146, 2005.

18. KAWABATA, G. y cols.: "Laparoscopic radical prostatectomy: initial 17 case report". Nippon Hinyokika Gakkai Zasshi, 92: 647, 2001.

19. JACOB, F. y cols.: "Laparoscopic radical prostatectomy: preliminary results". Eur. Urol., 37: 615, 2000.

20. HATTORI, R. y cols.: "Laparoscopic radical prostatectomy-initial 10 cases". Nippon Hinyokika Gakkai Zasshi, 92: 603, 2001.

21. HABUCHI, T., y cols.: "Early results in the initial 15 cases of laparoscopic radical prostatectomy in Akita University Medical Center”. Hinyokika Kiyo, 48: 139, 2002.

22. SALOMON, L., y cols.: "Open versus laparoscopic radical prostatectomy: Part II". BJU Int., 94: 244, 2004.

23. TURK, I. y cols.: "Laparoscopic radical prostatectomy. Technical aspects and experience with 125 cases". Eur. Urol., 40: 46, 2001.

24. MENON, M., y cols.: "Vattikuti institute prostatectomy: contemporary technique and analysis of results". Eur. Urol., 51: 648, 2007.

25. SIM, H.G. y cols.: "Team-based approach reduces learning curve in robot-assisted laparoscopic radical prostatectomy". Int. J. Urol., 13: 560, 2006.

26. MENON, M. y cols.: "Prospective comparison of radical retropubic prostatectomy and robot-assisted anatomic prostatectomy: the Vattikuti Urology Institute experience". Urology, 60: 864, 2002.

27. FARNHAM, S.B. y cols.: "Intraoperative blood loss and transfusion requirements for robotic-assisted radical prostatectomy versus radical retropubic prostatectomy". Urology, 67: 360, 2006.

28. SMITH, J.A., Jr.: "Robotically assisted laparoscopic prostatectomy: an assessment of its contemporary role in the surgical management of localized prostate cancer". Am. J. Surg., 188: 63, 2004.

29. CURTO, F. y cols.: "Nerve sparing laparoscopic radical prostatectomy: our technique". Eur. Urol., 49: 344, 2006.

30. SULTAN, R. y cols.: "Time to return to work and physical activity following open radical retropubic prostatectomy". J. Urol., 176: 1420, 2006.

31. RASSWEILER, J. y cols.: "Laparoscopic and robotic assisted radical prostatectomy - critical analysis of the results". Eur. Urol., 49: 612, 2006.

32. GUILLONNEAU, B. y cols.: "Laparoscopic radical prostatectomy: technical and early oncological assessment of 40 operations". Eur. Urol., 36: 14, 1999.

33. CATHELINEAU, X.; ROZET, F.; VALLANCIEN, G.: "Robotic radical prostatectomy: the European experience". Urol. Clin. North Am., 31: 693, 2004.

34. WALSH, P.C.: Anatomic radical retropubic prostatectomy". Campbell's Urology, P.C. Walsh, Retik B, Vaughan ED, Wein AJ, Editor. WB Saunders: Philadelphia. p. 2565-2588, 1998.

35. NADU, A. y cols.: "Early removal of the catheter after laparoscopic radical prostatectomy". J. Urol., 166: 1662, 2001.

36. BHAYANI, S.B. y cols.: "Laparoscopic radical prostatectomy: a multi-institutional study of conversion to open surgery". Urology, 63: 99, 2004.

37. WEBSTER, T.M. y cols.: "Robotic assisted laparoscopic radical prostatectomy versus retropubic radical prostatectomy: a prospective assessment of postoperative pain". J. Urol., 174: 912, 2005.

38. HOLZBEIERLEIN, J.M.; SMITH, J.A.: "Radical prostatectomy and collaborative care pathways". Semin. Urol. Oncol., 18: 60, 2000.

39. BIANCO, F.J. Jr.; SCARDINO, P.T.; EASTHAM, J.A.: "Radical prostatectomy: long-term cancer control and recovery of sexual and urinary function ("trifecta")". Urology, 66: 83, 2005.

40. STEPHENSON, A.J. y cols.: "Postoperative nomogram predicting the 10 -year probability of prostate cancer recurrence after radical prostatectomy". J. Clin. Oncol., 23: 7005, 2005.

41. SWINDLE, P. y cols.: "Do margins matter? The prognostic significance of positive surgical margins in radical prostatectomy specimens". J. Urol., 174: 903, 2005

42. WIEDER, J.A.; SOLOWAY, M.S.: "Incidence, etiology, location, prevention and treatment of positive surgical margins after radical prostatectomy for prostate cancer". J. Urol., 160: 299, 1998.

43. OHORI, M.; SCARDINO, P.T.: "Localized prostate cancer". Curr. Probl. Surg., 39: 833, 2002.

44. BLUTE, M.L. y cols.: "Use of Gleason score, pros- 
tate specific antigen, seminal vesicle and margin status to predict biochemical failure after radical prostatectomy". J. Urol., 165: 119, 2001.

45. BROWN, J.A. y cols.: "Transperitoneal versus extraperitoneal approach to laparoscopic radical prostatectomy: an assessment of 156 cases". Urology, 65: 320, 2005.

46. HUMPHREYS, M.R. y cols.: "Minimally invasive radical prostatectomy". Mayo Clin. Proc., 79: 1169, 2004.

47. ZINCKE, H. y cols.: "Radical prostatectomy for clinically localized prostate cancer: long-term results of 1,143 patients from a single institution". J. Clin. Oncol., 12: 2254, 1994.

48. WARD, J.F. y cols.: "Radical prostatectomy for clinically advanced (cT3) prostate cancer since the advent of prostate-specific antigen testing: 15-year outcome". BJU Int., 95: 751, 2005.

49. MENON, M.; TEWARI, A.; PEABODY, J.: "Vattikuti Institute prostatectomy: technique". J. Urol., 169: 2289, 2003.

50. PARTIN, A.W. y cols.: "Serum PSA after anatomic radical prostatectomy. The Johns Hopkins experience after 10 years". Urol. Clin. North Am., 20: 713, 1993.

51. SALOMON, L. y cols.: "Outcome and complications of radical prostatectomy in patients with PSA $<10 \mathrm{ng} / \mathrm{ml}$ : comparison between the retropubic, perineal and laparoscopic approach". Prostate Cancer Prostatic Dis., 5: 285, 2002.

52. HOZNEK, A. y cols.: "Laparoscopic radical prostatectomy. The Creteil experience". Eur. Urol., 40: 38, 2001.

53. AHLERING, T.E. y cols.: "Robotic radical prostatectomy: a technique to reduce pT2 positive margins". Urology, 64: 1224, 2004.

54. GETTMAN, M.T.; BLUTE, M.L.: "Critical comparison of laparoscopic, robotic, and open radical prostatectomy: techniques, outcomes, and cost". Curr. Urol. Rep., 7: 193, 2006.

55. GUILLONNEAU, B. y cols.: "Laparoscopic radical prostatectomy: oncological evaluation after 1,000 cases a Montsouris Institute". J. Urol., 169: 1261, 2003.

56. ROZET, F. y cols.: "Extraperitoneal laparoscopic radical prostatectomy: a prospective evaluation of 600 cases". J. Urol., 174: 908, 2005.

57. PATEL, V.R. y cols.: "Robotic radical prostatectomy in the community setting--the learning curve and beyond: initial 200 cases". J. Urol., 174: 269, 2005.

58. MIKHAIL, A.A. y cols.: "Robotic-assisted laparoscopic prostatectomy: first 100 patients with one year of follow-up". Urology, 68: 1275, 2006.

59. HSU, E.I.; HONG, E.K.; LEPOR, H.: "Influence of body weight and prostate volume on intraoperative, perioperative, and postoperative outcomes after radical retropubic prostatectomy". Urology, 61: 601, 2003.
60. SALOMON, L. y cols.: "Radical prostatectomy by the retropubic, perineal and laparoscopic approach: 12 years of experience in one center". Eur. Urol., 42: 104, 2002.

61. LERNER, S.E. y cols.: "Morbidity of contemporary radical retropubic prostatectomy for localized prostate cancer”. Oncology (Williston Park), 9: 379, 1995.

62. GUILLONNEAU, B. y cols.: "Laparoscopic radical prostatectomy: assessment after 240 procedures". Urol. Clin. North Am., 28: 189, 2001.

63. RASSWEILER, J. y cols.: "Laparoscopic versus open radical prostatectomy: a comparative study at a single institution”. J. Urol., 169: 1689, 2003.

64. MENON, M.; HEMAL, A.K.: "Vattikuti Institute prostatectomy: a technique of robotic radical prostatectomy: experience in more than 1000 cases". J. Endourol., 18: 611, 2004.

65. HU, J.C. y cols.: "Perioperative complications of laparoscopic and robotic assisted laparoscopic radical prostatectomy". J. Urol., 175: 541, 2006.

66. JOSEPH, J.V. y cols.: "Robotic extraperitoneal radical prostatectomy: an alternative approach". J. Urol., 175: 945, 2006.

67. VAN APPLEDORN, S. y cols.: "Robotic laparoscopic radical prostatectomy: setup and procedural techniques after 150 cases". Urology, 67: 364, 2006.

68. CATALONA, W.J. y cols.: "Potency, continence and complication rates in 1,870 consecutive radical retropubic prostatectomies". J. Urol., 162: 433, 1999.

69. RASSWEILER, J. y cols.: "Heilbronn laparoscopic radical prostatectomy. Technique and results after 100 cases". Eur. Urol., 40: 54, 2001.

70. VALLANCIEN, G. y cols.: "Complications of transperitoneal laparoscopic surgery in urology: review of 1,311 procedures at a single center". J. Urol., 168: 23, 2002.

71. GUILLONNEAU, B.; VALLANCIEN, G.: "Laparoscopic radical prostatectomy: the Montsouris technique". J. Urol., 163: 1643, 2000.

72. CATALONA, W.J.; SMITH, D.S.: "Cancer recurrence and survival rates after anatomic radical retropubic prostatectomy for prostate cancer: intermediate-term results". J. Urol., 160: 2428, 1998.

73. HULAND, H.: "Radical prostatectomy: options and issues". Eur. Urol., 39: 3, 2001.

74. LEPOR, H.; KACI, L.: "Contemporary evaluation of operative parameters and complications related to open radical retropubic prostatectomy". Urology, 62: 702, 2003.

75. RASSWEILER, J. y cols.: "Laparoscopic radical prostatectomy with the Heilbronn technique: oncological results in the first 500 patients". J. Urol., 173: 761, 2005.

76. CHIEN, G.W. y cols.: "Modified clipless antegrade nerve preservation in robotic-assisted laparoscopic radical prostatectomy with validated sexual function evaluation”. Urology, 66: 419, 2005. 